ACCEPTED MANUSCRIPT

\title{
Proton range verification with ultrasound imaging using injectable radiation sensitive nanodroplets: a feasibility study
}

To cite this article before publication: Bram Carlier et al 2020 Phys. Med. Biol. in press https://doi.org/10.1088/1361-6560/ab7506

\section{Manuscript version: Accepted Manuscript}

Accepted Manuscript is "the version of the article accepted for publication including all changes made as a result of the peer review process, and which may also include the addition to the article by IOP Publishing of a header, an article ID, a cover sheet and/or an 'Accepted

Manuscript' watermark, but excluding any other editing, typesetting or other changes made by IOP Publishing and/or its licensors"

This Accepted Manuscript is $\odot 2020$ Institute of Physics and Engineering in Medicine.

During the embargo period (the 12 month period from the publication of the Version of Record of this article), the Accepted Manuscript is fully protected by copyright and cannot be reused or reposted elsewhere.

As the Version of Record of this article is going to be / has been published on a subscription basis, this Accepted Manuscript is available for reuse under a CC BY-NC-ND 3.0 licence after the 12 month embargo period.

After the embargo period, everyone is permitted to use copy and redistribute this article for non-commercial purposes only, provided that they adhere to all the terms of the licence https://creativecommons.org/licences/by-nc-nd/3.0

Although reasonable endeavours have been taken to obtain all necessary permissions from third parties to include their copyrighted content within this article, their full citation and copyright line may not be present in this Accepted Manuscript version. Before using any content from this article, please refer to the Version of Record on IOPscience once published for full citation and copyright details, as permissions will likely be required. All third party content is fully copyright protected, unless specifically stated otherwise in the figure caption in the Version of Record.

View the article online for updates and enhancements. 


\title{
Proton range verification with ultrasound imaging using injectable radiation sensitive nanodroplets: a feasibility study
}

\author{
Bram Carlier ${ }^{1,2,3}$, Sophie V. Heymans ${ }^{1,4}$, Sjoerd Nooijens ${ }^{5}$, Yosra Toumia ${ }^{6}$, Marcus \\ Ingram ${ }^{5}$, Gaio Paradossi ${ }^{6}$, Emiliano d'Agostino ${ }^{7}$, Uwe Himmelreich ${ }^{3,8}$, Jan D'hooge $^{5}$, \\ Koen Van Den Abeele ${ }^{4}$ and Edmond Sterpin ${ }^{2,3}$
}

${ }^{1}$ These two authors contributed equally

${ }^{2}$ Department of Oncology, KU Leuven, Leuven, Belgium

${ }^{3}$ Molecular Small Animal Imaging Center, KU Leuven, Leuven, Belgium ${ }^{4}$ Department of Physics, KU Leuven Campus Kulak, Kortrijk, Belgium ${ }^{5}$ Department of Cardiovascular Sciences, KU Leuven, Leuven, Belgium ${ }^{6}$ Department of Chemical Sciences and Technology, University of Rome Tor Vergata, Rome, Italy

${ }^{7}$ DoseVue, Philips Open Manufacturing Campus, Turnhout, Belgium

${ }^{8}$ Department of Imaging \& Pathology, KU Leuven, Leuven, Belgium

E-mail: edmond.sterpin@kuleuven.be

\begin{abstract}
Technologies enabling in vivo range verification during proton therapy are actively sought as a means to reduce the clinical safety margins currently adopted to avoid tumor underdosage. In this contribution, we applied the semi-empirical theory of radiation-induced vaporization of superheated liquids to coated nanodroplets. Nanodroplets are injectable phase-change contrast agents that can vaporize into highly echogenic microbubbles to provide contrast in ultrasound images. We exposed nanodroplet dispersions in aqueous phantoms to monoenergetic proton beams of varying energies and doses. Ultrasound imaging of the phantoms revealed that radiation-induced droplet vaporization occurred in regions proximal to the proton Bragg peak. A statistically significant increase in contrast was observed in irradiated regions for doses as low as $2 \mathrm{~Gy}$ and found to be proportional to the proton fluence. The absence of enhanced response in the vicinity of the Bragg peak, combined with theoretical considerations, suggest that droplet vaporization is induced by high linear energy transfer (LET) recoil ions produced by nuclear reactions with incoming protons. Vaporization profiles were compared to non-elastic cross sections and LET characteristics of oxygen recoils. Shifts between the ultrasound image contrast drop and the expected proton range showed a sub-millimeter reproducibility. These early findings confirm the potential of superheated nanodroplets as a novel tool for proton range verification.
\end{abstract}

Keywords: proton therapy, range verification, dosimetry, ultrasound, nanodroplets

\section{Introduction}

The increasingly growing fleet of proton therapy facilities, owing to substantial cost reduction and compactness improvements over the past ten years, has contributed in making proton therapy accessible to a variety of clinical indications (Thariat et al 2013). The favorable depth dose distribution of protons implies that excellent dose conformality and therefore healthy tissue sparing could be achieved. However, taking full advantage of the physical selectivity of protons, e.g. to preserve organs-at-risk, is 
hampered by uncertainties in their in vivo range, which arise from inaccuracies of the stopping power calculation from computed tomography (CT) scans, imaging artifacts, setup errors, patient motion and anatomical changes throughout the treatment (Paganetti 2012, Knopf and Lomax 2013). Additionally, to avoid tumor underdosage due to these range uncertainties, considerable safety margins (up to several millimeters), conservative planning strategies (sub-optimal choice of beam angles), or a substantial value of the range uncertainty parameter used in robust optimizers (around 3\%) have been adopted in most proton therapy facilities (Paganetti 2012). In order to reduce these margins and gather more insight into the influence of different factors on the range, an urgent need for accurate in vivo range verification techniques exists.

Several methods for in vivo range verification have been proposed, however, none of them are routinely employed in the clinic. The most investigated technology relies on positron emission tomography (PET) imaging of positron-emitting isotopes activated by proton nuclear interactions (Paganetti 2012). The measured activity distribution is correlated with the actual proton range through Monte Carlo simulations. PET imaging can be performed offline (Parodi et al 2007), in-room (Min et al 2013) or in-beam (Kraan et al 2014, Helmbrecht et al 2012). While in-room and in-beam acquisitions benefit from shorter scan times and higher resolution compared to offline PET imaging, they require bespoke detectors and may affect the throughput in the treatment room (Paganetti 2019). Alternatively, prompt gamma imaging (PGI) makes use of gamma rays emitted by nuclei excited by the incoming proton beam for real-time range verification (Jongen and Stichelbaut 2003, Min et al 2006). PGI has been recently tested to assess range shifts in a patient with brain cancer and demonstrated a shift retrieval precision of $2 \mathrm{~mm}$ (Xie et al 2017), but its translation towards clinical applications is hampered by technological limitations and detector cost (Knopf and Lomax 2013, Rohling et al 2017). Range probe (1D) and proton radiography $(2 \mathrm{D} / 3 \mathrm{D})$ require high energy protons completely traversing the body in the low-dose plateau (Knopf and Lomax 2013, Mumot et al 2010, Plautz et al 2014). The stopping power of the body tracks is then determined from the residual ranges of the transmitted protons. However, typical proton energies used cannot ensure the beam traverses the patient at all beam angles, especially in the abdomen (Paganetti 2019).

Detection of ionizing radiation by means of the vaporization of superheated droplets, i.e. metastable droplets operated at a temperature above their boiling point, was achieved in the 1950s by Donald Glaser (Glaser 1952). Since then, the use of superheated emulsions significantly expanded to different fields such as space applications, medical physics, neutron dosimetry or dark matter search (Roy 2001). These detectors typically feature superheated drops of dimensions ranging from tens to thousands of microns, embedded in a compliant polymeric or aqueous matrix (D'Errico 2001). Upon exposure to radiation, the drops vaporize into bubbles which can be detected either by visual inspection, volumetric measurement or acoustic readout (Sarkar et al 2006, D'Errico 2001). Two decades ago, Apfel envisioned the use of injectable superheated emulsions as in vivo dosimeter (Apfel 1998), but to our knowledge, the idea has never been pursued.

Over the past ten years, nanodroplets, or phase-change contrast agents (PCCAs), have become increasingly popular as versatile contrast agents for ultrasound imaging and therapy (Sheeran and Dayton 2012). They consist of a perfluorocarbon liquid core surrounded by a stabilizing lipidic or polymeric shell, whose diameter typically ranges from hundreds of nanometers to a few microns (Lea-Banks et al 2019). Nanodroplets can be injected intravenously and circulate inside the patient's vasculature, where the smallest sizes $(<200 \mathrm{~nm})$ are able to extravasate. Additionally, the shell can be functionalized to target tissues of interest, making them suitable for molecular imaging and targeted therapy (Deshpande et al 2010). Localized nanodroplet vaporization can be achieved with ultrasound waves at moderate to high intensities (Sheeran et al 2012, Toumia et al 2019) or through laser heating (Dove et al 2014), yielding micrometer-sized echogenic bubbles readily imaged with Contrast Enhanced Ultrasound Imaging (CEUS). In order to minimize potential tissue damage from cavitation or heating, droplet vaporization should be achieved with moderate levels of acoustic or thermal energy (Sheeran et al 2011). Therefore, the droplet liquid core is generally designed to be in a metastable superheated state.

Here, the applicability of the radiation-induced nucleation theory to submicron-sized superheated droplets is evaluated. Specifically, we upgraded the naked superheated emulsions used in dosimetry into injectable nanodroplets similar to the ones employed for CEUS. First, we give a brief overview of the generally accepted theory of superheated droplet vaporization induced by ionizing radiation. Then, we demonstrate the existence of a radiation response and assess the suitability of these radiation sensors for proton range verification. To this aim, tissue-mimicking phantoms with entrapped nanodroplets were irradiated with varying proton energies and the relationship between the resulting ultrasound signals and the predicted proton range was investigated. Finally, the potential dose sensitivity was examined and the feasibility of the presented approach at clinically-relevant doses is shown.

\section{Materials and methods}

\subsection{Theory of nucleation induced by ionizing radiation}

The nucleation of bubbles along particle tracks in a metastable liquid is a complex physics problem involving time and length scales spanning several orders of magnitude and 
different fields such as thermodynamics and radiation physics, for which a complete analytical description is still lacking. The most widely accepted semi-empirical model combines the thermal spike theory developed by Seitz (Seitz 1958) with the isothermal spontaneous nucleation thermodynamics. In Seitz's theory, the kinetic energy of charged particles is transferred to the medium by a multitude of highly localized temperature spikes forming along their track (West 1998, Apfel 1979). The thermal spikes occur within a time scale so small compared to thermal diffusion that the liquid explodes into vapor embryos (D’Errico 2001, Sarkar et al 2006) along the particle track. These vapor embryos then combine and form spherical bubbles, which can grow indefinitely provided that the initial bubble size exceeds a critical radius, determined from the thermodynamics of phase equilibrium,

$$
R_{c}=\frac{2 \sigma}{\left(p_{s}-p_{l}\right)\left(1-\rho_{v} / \rho_{l}\right)}
$$

where $\sigma$ is the surface tension of the superheated liquid, $p_{s}$ is the saturation pressure, $p_{l}$ is the pressure inside the superheated liquid drop, and $\rho_{v}$ and $\rho_{l}$ are the densities of the vapor and liquid phases, respectively.

The energy required to nucleate a critical vapor bubble is obtained from homogeneous nucleation theory, with additional terms specific to radiation-induced nucleation (D’Errico 2001).

$$
\begin{gathered}
W_{t o t}=\frac{16 \pi \sigma^{3}}{3\left(p_{s}-p_{l}\right)^{2}\left(1-\rho_{v} / \rho_{l}\right)^{2}} \\
\times\left[1+\frac{2 \Delta H}{\left(p_{s}-p_{l}\right)\left(1 / \rho_{v}-1 / \rho_{l}\right)}-3 \frac{T \mathrm{~d} \sigma}{\sigma} \frac{\mathrm{d} T}{}\right]+W_{i r r} \\
W_{i r r}=2 \pi \rho_{l} R_{c}^{3} \dot{R}^{2} \\
\dot{R}=\frac{4 D\left(\rho_{l} / \rho_{v}\right)^{1 / 3}}{R_{c}} \\
D=\frac{k}{\rho_{l} c_{p}}
\end{gathered}
$$

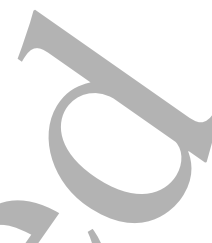

where $\Delta H$ is the latent vaporization heat of the fluid, and $W_{i r}$ accounts for the irreversible energy losses from the action of viscous forces and the transfer of kinetic energy to the surrounding liquid (D’Errico 2001). $\dot{R}$ is the vapor wall velocity, $D$ is the thermal diffusivity of the liquid, and $k$ and $c_{p}$ are its thermal conductivity and specific heat, respectively.

In order to nucleate a vapor bubble of dimensions larger than the critical radius, the energy deposited by the charged particle along an effective path length $\left(L_{e f f}\right)$ must exceed $W_{t o t}$ (D'Errico 1999). The effective path length is often assumed proportional to the critical radius, yielding $L_{\text {eff }}=a R_{c}$. However, a single value of the proportionality constant $(a)$, also called nucleation parameter, is insufficient to describe the behavior of superheated drop detectors for all degrees of superheat (D'Errico 1999). Moreover, the linear relationship between the effective length and the critical radius remains questionable (Andrews et al 2006). Nevertheless, most authors assume a constant value of the nucleation parameter that typically ranges from 2 to 13 (Ing et al 1997).

The energy transferred by a charged particle per unit track length in a medium is given by its linear energy transfer (LET) (Paganetti 2019). The nucleation condition is given by:

$$
\int_{0}^{L_{e f f}} \frac{\mathrm{d} E}{\mathrm{~d} x} \mathrm{~d} x \geq W_{\text {tot }}
$$

and can be further expressed in terms of track-averaged LET (Ing et al 1997):

$$
\left\langle\frac{\mathrm{d} E}{\mathrm{~d} x}\right\rangle_{L_{\text {eff }}} \geq \frac{W_{\text {tot }}}{a R_{c}}
$$

The left hand side of the equation is only dependent on the energy deposition characteristics of the radiation, while the right hand side obeys the thermodynamic properties of the superheated fluid. Since the nucleation energy drops when the superheat increases, the LET threshold of superheated drop detectors is inversely proportional to the degree of superheat.

The "reduced superheat" parameter is commonly used to describe the operating point of a superheated liquid with respect to the temperature boundaries of the superheated state (D’Errico 1999), and is defined as:

$$
s=\frac{T-T_{b}}{T_{c}-T_{b}}
$$

$T_{b}$ is the boiling temperature at atmospheric pressure, and $T_{c}$ is the critical temperature of the fluid above which the liquid phase can no longer exist. By appropriately tuning the degree of superheat of the liquid core, one can tailor the droplet sensitivity to different types of radiation. Typically, neutron dosimeters operate at $s=0.2$, as the droplets are vaporized by high-LET secondary charged particles produced by nuclear reactions (D'Errico 2001). To sensitize bubble detectors to low-LET radiation such as photons and protons, higher degrees of superheat are required, which comes at the cost of decreased droplet stability. The practical limit of superheat is reached for $s=0.65$, when the metastable liquid spontaneously vaporizes (Porteous and Blander 1975, D'Errico et al 2000). Detection of proton radiation in the vicinity of the Bragg peak with superheated drops was reported by several groups (Green et al 2005, D'Errico and Egger 1994, Guo et al 2002), indicating that the threshold for proton detection lies between $s=0.35$ and $s=0.42$, corresponding to an LET threshold of $70-90 \mathrm{keV} / \mu \mathrm{m}$, typically reached by protons at the end of their range. Proton irradiation of bubble detectors with lower degrees of superheat revealed that high-LET nuclear reaction products (heavy recoils) induce uniform vaporization tracks (Green et al 2005, D'Errico et al 1997, Miller et al 2018, Takada et al 2004). 


\subsection{Nanodroplet synthesis and characterization}

2.2.1 Nanodroplet composition and synthesis. The nanodroplets employed in this study are comprised of a perfluorobutane $\left(\mathrm{C}_{4} \mathrm{~F}_{10}\right.$, boiling point of $\left.-2^{\circ} \mathrm{C}\right)$ liquid core encapsulated by a polymerizable fatty acid monolayer of 10,12-pentacosadiynoic acid (PCDA). The complete nanodroplet synthesis is described elsewhere (Toumia et al 2019) and can be performed with standard laboratory equipment. Moreover, feasibility of production was observed to be reproducible and laboratory-independent, as nanodroplets were initially developed in the University of Rome Tor Vergata, and subsequently prepared in the KU Leuven campus KULAK. In brief, decafluorobutane was fluxed for a few seconds into an empty glass vial sealed with a rubber septum and immersed in liquid nitrogen to ensure liquefaction. Afterwards, injection of $6 \mathrm{ml}$ of PCDA aqueous suspension ( $1 \mathrm{mM})$ in the vial, followed by a 10-minute sonication in an ice-cold ultrasound bath, yielded a milky suspension of nanodroplets. Non-encapsulated decafluorobutane vaporized during sonication, filling the headspace of the glass vial. After addition of surfactant (Pluronic F127) and photoinitiator solutions (Irgacure 2959), nanodroplets were exposed to $352 \mathrm{~nm}$ UV-light (UV lamp model ENF-260C, Spectroline Corporation, Westbury, NY) for 30 minutes, to polymerize the PCDA shell through the diacetylene moieties, resulting in formation of a blue color and enhanced nanodroplet stability. Finally, the vial was stored at $4^{\circ} \mathrm{C}$ for three days before use, and the droplets were washed with distilled water by centrifugation (1000 g-force, 6 minutes) prior to phantom preparation.

\subsubsection{Nanodroplet size and concentration. The size} distribution of the nanodroplets was measured by Dynamic Light Scattering (DLS), as described in (Toumia et al 2019). The median diameter of the nanodroplets (intensity-weighted) was found to be $842 \mathrm{~nm} \pm 12 \mathrm{~nm}(\mathrm{n}=4)$ and the polydispersity index was $0.25 \pm 0.02$. The concentration of nanodroplets was evaluated using ${ }^{19} \mathrm{~F}$ NMR spectroscopy (400 MHz Avance II, Bruker Biospin GmbH, Rheinstetten, Germany) referenced against $5 \mathrm{mM}$ fluorocytosine.

\subsection{Phantom synthesis}

Gelatin was chosen to fix the droplets as it has a low gelling temperature and excellent tissue-mimicking ultrasonic properties (Culjat et al 2010). The gelatin powder (6\% vol., ITW Reagents) was added to deionized water at room temperature to prevent flocculation and then boiled to ensure complete dissolution and removal of entrapped air bubbles. Afterwards, the mixture was poured in rectangular phantom containers (inner dimensions: length $=54 \mathrm{~mm}$, width $=26 \mathrm{~mm}$, depth $=31 \mathrm{~mm}$, $43.5 \mathrm{ml}$ in volume, figure 1). Different volumes of nanodroplets were added (see table 1) using an $18 \mathrm{G}$ needle when the gelatin solution reached $32^{\circ} \mathrm{C}$ to reduce spontaneous
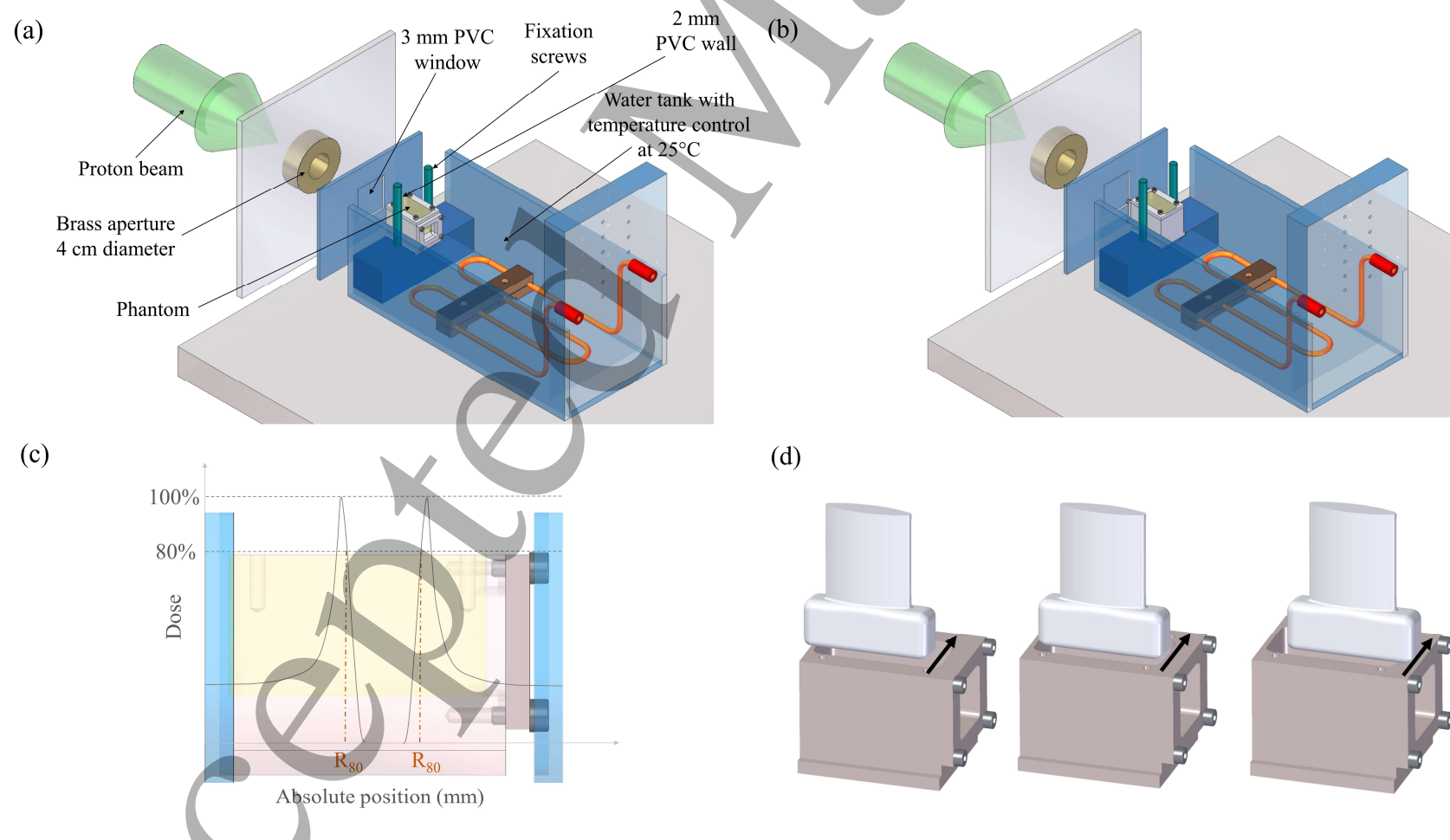

(d)

Figure 1. Schematics of the irradiation setup, for phantoms in forward (a) and reverse position (b). (c) Position of the Bragg peaks inside the gelatin phantoms. (d) Imaging of the phantoms with an ultrasound probe on three different positions to cover the entire phantom length. The lateral ultrasound axis is parallel to the direction of the proton beam, and the axial ultrasound axis corresponds to the phantom depth. Eleven images were acquired at each position by moving the ultrasound probe across the phantom width (elevational axis).
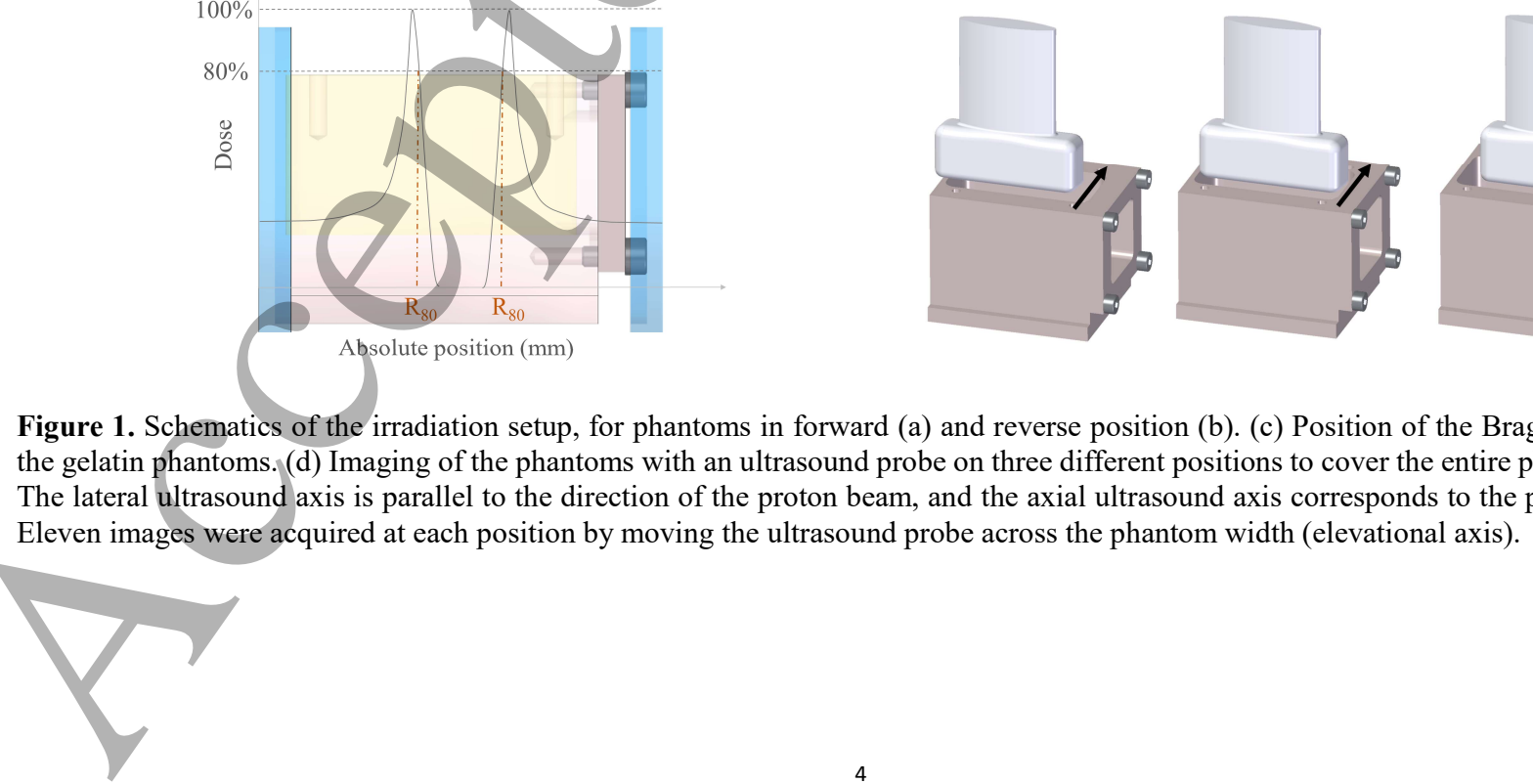
vaporization due to large injection pressure and temperature fluctuations. After manual homogenization, phantoms were quickly cooled on ice to minimize nanodroplet sedimentation during solidification. Due to the observed limited stability of the nanodroplets in gelatin over time (figure 2), the phantoms were always made less than three hours before irradiation.

\subsection{Irradiation protocol}

2.4.1 Irradiation setup. Proton irradiation was carried out at the Centre de Ressources du Cyclotron (UCLouvain, Louvain-la-Neuve, Belgium), an experimental research facility. The cyclotron (CYCLONE 110) produced a monoenergetic, passively scattered proton beam at $62 \mathrm{MeV}$. The proton range was modulated in discrete steps by inserting different thicknesses of degrader material in front of the irradiated sample. A brass aperture of $40 \mathrm{~mm}$ diameter was positioned in front of the phantoms to limit the field size. The phantoms were fixed in a water tank heated to $25^{\circ} \mathrm{C}$ and equipped with temperature control (feedback provided by an immersed thermocouple, temperature accuracy of $\pm 0.5^{\circ} \mathrm{C}$ ), as illustrated in figure 1(ab). Each phantom was irradiated twice, first in the configuration of figure $1(\mathrm{a})$, and then the phantom was rotated by $180^{\circ}$ to irradiate the other side (figure 1(b)). Since the proton range was shorter than half of the phantom length, we assumed each irradiation independent of the other. In both configurations, the protons traveled a certain depth before penetrating the gelatin phantoms. Due to the asymmetric design of the phantom containers, the path traveled by the proton beam before penetrating the gelatin differed for the forward and reverse positions. This difference, as well as the presence of PVC material in the beam path (water tank entrance window and PVC phantom container walls), was accounted for when estimating the Bragg peak position in the phantom. The impact of a sub-millimeter thin acoustic window sheet on one side of the phantom container (figure 1) was assumed to be negligible.

2.4.2 Absolute range measurement. Two beam energies were employed during the experiments $(62 \mathrm{MeV}$ and 46.8

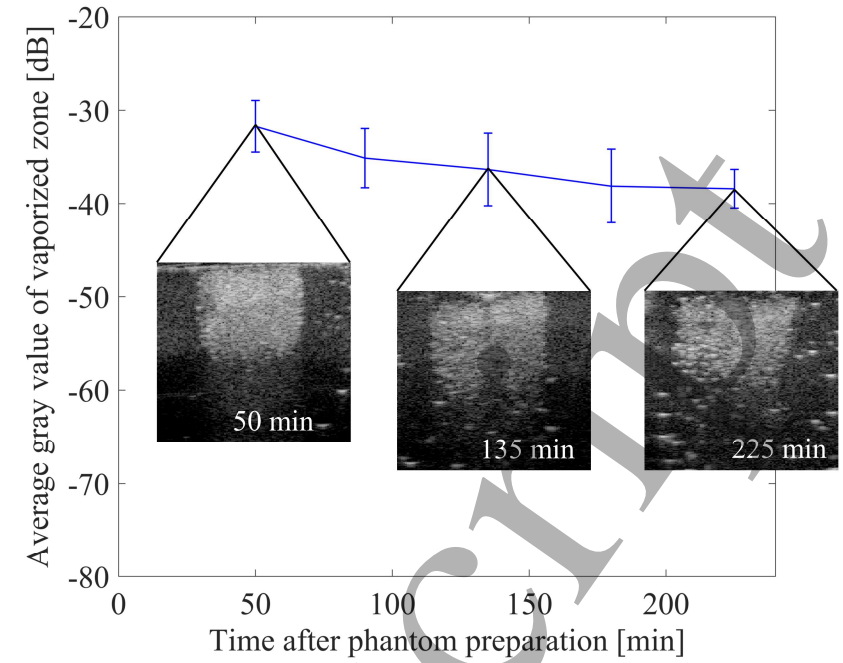

Figure 2. Nanodroplet vaporization potential over time (mean \pm standard deviation over five phantoms), represented as the average gray value in $16 \mathrm{~mm}^{2}$ regions of acoustically vaporized nanodroplets ( $25 \mu \mathrm{M}$ nanodroplets in $6 \%$ gelatin phantoms). Although densely packed microbubbles were generated at each time point, the slowly decreasing gray value indicated a limited stability of nanodroplets in gelatin over time.

$\mathrm{MeV}$ ). For each energy, we performed an absolute measurement of the proton range with a bespoke setup consisting of a water tank equipped with a thin $23 \mu \mathrm{m}$ polyethylene terephtalate entrance window. An automated 1D linear stage was employed to move a dosimetry diode $(1.33 \mathrm{~mm}$ water equivalent thickness (WET), model PR60020, PTW, Freiburg, Germany) along the depth of the proton beam with a step size of $1 \mathrm{~mm}$. The measured depth-dose curve was fitted to an analytical approximation of the Bragg profile (Bortfeld 1997) to determine the range (defined here as the distal $80 \%$ dose point, $R_{80}$, as recommended in (Paganetti 2012)) and the skin-to-peak dose ratio for the dose calculation. To account for the PVC layers of the water tank and phantom container, we measured the beam profile with and without a 5-mm thick PVC plate in front of the water tank. Relying on these range measurements and the phantom geometry, the $R_{80}$ values for both the forward and reverse positions were calculated (figure 1(c)).

Table 1. Irradiation conditions

\begin{tabular}{|c|c|c|c|c|c|c|c|c|}
\hline \multirow{2}{*}{$\begin{array}{l}\text { Phantom } \\
\text { composition }\end{array}$} & \multirow{2}{*}{$\begin{array}{l}\text { Number of } \\
\text { phantoms }\end{array}$} & \multicolumn{3}{|c|}{ Forward phantom position } & \multicolumn{3}{|c|}{ Reverse phantom position } & \multirow[b]{2}{*}{ Ultrasound imaging } \\
\hline & & $\begin{array}{l}\text { Dose } \\
{[\mathrm{Gy}]}\end{array}$ & $\begin{array}{l}\text { Dose rate } \\
{[\mathrm{Gy} / \mathrm{min}]}\end{array}$ & $\begin{array}{l}\text { Energy } \\
{[\mathrm{MeV}]}\end{array}$ & $\begin{array}{l}\text { Dose } \\
{[\mathrm{Gy}]}\end{array}$ & $\begin{array}{l}\text { Dose rate } \\
{[\mathrm{Gy} / \mathrm{min}]}\end{array}$ & $\begin{array}{l}\text { Energy } \\
{[\mathrm{MeV}]}\end{array}$ & \\
\hline $\begin{array}{l}\text { 6\% Gelatin } \\
\text { No NDs }\end{array}$ & & & 2 & 62 & 20 & 4 & 62 & $\begin{array}{l}\text { Pre-irradiation } \\
\text { Post-irradiation }\end{array}$ \\
\hline $\begin{array}{l}6 \% \text { Gelatin } \\
25 \mu \mathrm{M} \text { NDs }\end{array}$ & 3 & 10 & 2 & 62 & 20 & 4 & 62 & $\begin{array}{l}\text { Pre-irradiation } \\
\text { Post-irradiation }\end{array}$ \\
\hline $\begin{array}{l}6 \% \text { Gelatin } \\
25 \mu \mathrm{M} \text { NDs }\end{array}$ & & - & - & - & - & - & - & $\begin{array}{l}\text { Pre-immersion }\left(25^{\circ} \mathrm{C}\right) \\
\text { Post-immersion }\left(25^{\circ} \mathrm{C}\right)\end{array}$ \\
\hline $\begin{array}{l}6 \% \text { Gelatin } \\
50 \mu \mathrm{M} \text { NDs }\end{array}$ & 3 & 10 & 2 & 46.8 & 2 & 2 & 62 & Post-irradiation \\
\hline
\end{tabular}


2.4.3 Irradiation conditions. The irradiation conditions of each phantom are recorded in table 1 . Three phantoms with $25 \mu \mathrm{M}$ PCDA droplets were irradiated with $62 \mathrm{MeV}$ protons on both sides, $10 \mathrm{~Gy}$ in forward position and $20 \mathrm{~Gy}$ in reverse. A gelatin phantom without nanodroplets was also irradiated with the same parameters to verify that the gelatin matrix itself does not exhibit any dose response detectable via ultrasound imaging. Three phantoms with the same nanodroplet concentration were not irradiated and acted as controls. These phantoms were made simultaneously with their irradiated counterparts and immersed in a separate water tank at $25^{\circ} \mathrm{C}$ for ten minutes to mimic the thermal conditions of the irradiated phantoms. Additionally, three phantoms received a dose of 10 Gy at a different energy (46.8 MeV) in forward position, and a clinically-relevant dose of $2 \mathrm{~Gy}$ at $62 \mathrm{MeV}$ in reverse position. For these phantoms, the nanodroplet concentration was doubled. The reported doses and dose rates are evaluated at the Bragg peak, and dose calculations were performed using a peak-to-skin dose ratio equal to five, in agreement with the measured beam profiles. The proton entrance flux was measured by a calibrated ionization chamber present in the beam path.

\subsection{Ultrasound imaging}

Each phantom was immersed in water at room temperature and imaged with an experimental ultrasound scanner (DiPhAS, Fraunhofer IBMT, Germany) driving a 7.5 MHz linear array (L7-Xtech, Vermon, France). The lateral and axial resolution of the ultrasound system were evaluated as described elsewhere (Zhang et al 2018). Briefly, the full width at half maximum (FWHM) of the point spread function of an $80 \mu \mathrm{m}-$ diameter wire acting as point scatterer was determined at varying depths. This resulted in a representative lateral resolution of $1.18 \mathrm{~mm}$ and axial resolution of $0.29 \mathrm{~mm}$ along the depth of the phantom container.

The ultrasound probe was mounted on a manual 1D linear stage and moved to scan the phantom parallel to the proton beam direction, yielding up to 11 independent images per phantom (figure 1(d)). Because the probe's lateral field of view $(38.5 \mathrm{~mm})$ was smaller than the sensitive zone $(54 \mathrm{~mm})$, we acquired three different views of each phantom (figure 1(d)) aligned with either end or the center of the gelatin. Low pressure, plane wave imaging was employed, which was verified not to cause acoustic droplet vaporization. All phantoms except the last three phantoms in table 1 (due to time constraints) were imaged twice, before and after forward and reverse irradiation (or immersion in $25^{\circ} \mathrm{C}$ water tank for controls), with identical ultrasound parameters.

\subsection{Image processing}

2.6.1 Bubble localization and counting. Ultrasound images of the phantoms were divided in three zones: the region irradiated in the forward direction, the one irradiated in the reverse direction, and the space in between, distal to both forward and reverse irradiations (figure 1(c)). For each zone, a rectangular isometric region of interest (ROI) was defined, with a size of $7 \mathrm{~mm}$ parallel to the proton beam and $17 \mathrm{~mm}$ parallel to the phantom depth. These ROI sizes maximized bubble counts, while still ensuring complete containment in the respective zone and preventing artifacts from phantom wall reflections as well as irregularities at the gelatin surface. Bubbles were counted with an in-house developed algorithm, based on peak detection and thresholding on the pixel gray value. Bubbles were localized as the position of the brightest pixel of their point spread function, resulting in a lateral localization precision of $0.25 \mathrm{~mm}$ (one pixel). This approach showed sub-pixel agreement with the intensity-weighted center of mass calculation (Viessmann et al 2013) for the 80 $\mu \mathrm{m}$-diameter wire scatterer and was expected to provide an improved robustness in dense bubble zones. Additionally, an ellipsoidal inclusion zone was defined around each detected microbubble, with dimensions defined by the previously described FWHMs in the respective axial and lateral direction. If multiple microbubbles were detected in this zone, they were assumed to be originating from a single microbubble with the average position as its center. Depth-dependent thresholding was used to counteract the decrease of the mean gray value due to attenuation of the ultrasound wave (especially in regions with high bubble density).

2.6.2 Bubble count profiles. Bubble count profiles along the proton beam path were derived from the ultrasound images as illustrated in figure 3. Each microbubble detected by the bubble-counting algorithm (figure 3(a)) was assigned to a single pixel (brightest spot). Then, the bubble positions in the 11 frames per phantom view were combined (figure 3(b)). All bubbles in a rectangular bin of dimensions equal to the ROI depth in the depth direction and seven pixels in the lateral direction were summed and the resulting count was assigned to the central lateral pixel position. This ensured sufficient count statistics and smoothed out strong fluctuations, while preventing loss of profile information. Consecutively, the vertical bin was moved pixelwise along the lateral axis (parallel to the proton beam direction, see figure 3(c)) and the resulting lateral intensity profile was divided by the number of frames $(n=11)$. To provide full coverage of the lateral view, bubble count profiles derived from the three different probe positions were aligned and combined (figure 3(d)). Finally, to translate the pixel position into an absolute position in the phantom, the middle of the non-vaporized zone in the phantoms (figure 3(f)), defined as the center point between the two $50 \%$ drops in bubble count (figure 3(e)), was aligned with the position equidistant from the forward and reverse range $\left(R_{80}\right)$ values (figure 3(f)). The exact position of the $50 \%$ drop was determined as follows. First, the profiles were smoothed to decrease the impact of bubble count fluctuations. Then, the 
(a)

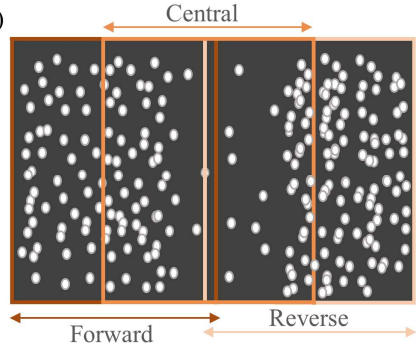

(b)

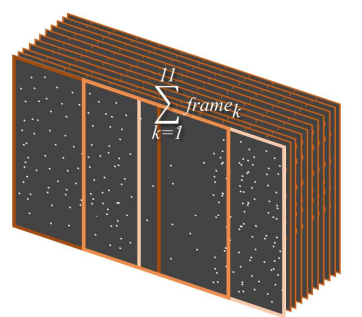

(c)

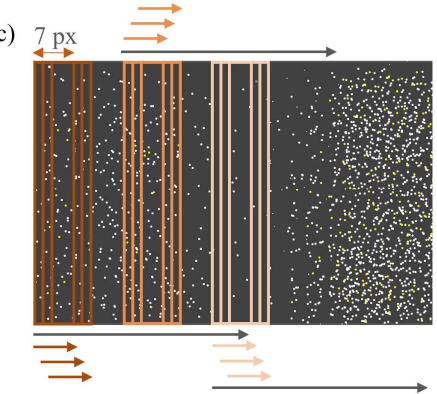

(d)

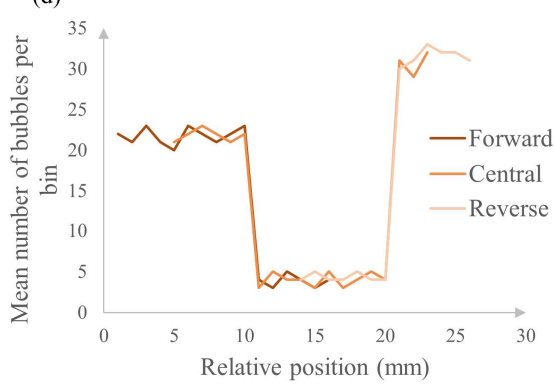

(e)

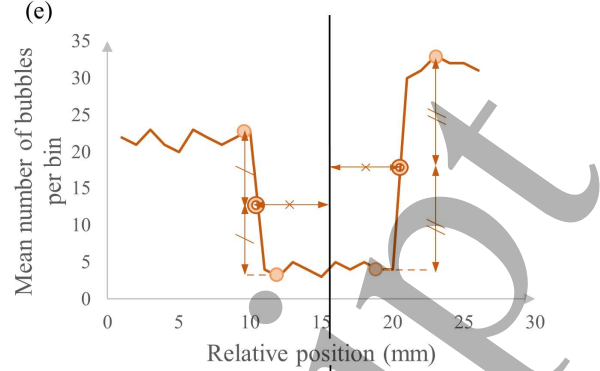

(f)

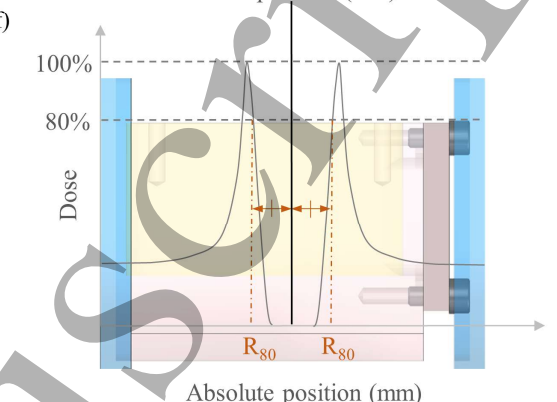

Figure 3. Schematic overview of the image processing. (a) Bubbles were identified in every frame of each view (forward, reverse, central) and their central position was stored. (b) For each view, all frames were combined. (c) Then, bubbles were counted in rectangular bins (width of seven pixels) and divided by the number of frames. The resulting bubble count was assigned to the central lateral position of the bin. Afterwards, the bin was moved pixelwise across the image's lateral axis to obtain a full bubble count profile. (d) The obtained bubble count profiles for the three views were aligned by template matching and combined by a weighted average to obtain a single profile across the entire phantom length. (e) The position of 50\% drop in bubble count was identified in the forward and reverse transition zones. (f) The midpoint between the two transition zones was aligned with the midpoint between the forward and reverse $R_{80}$ positions in the phantom to enable comparison with the proton range.

position corresponding to the peak value of the square of the first differential of the bubble count profile was detected. In a $10 \mathrm{~mm}$-interval around this position, the minimum and maximum bubble count was determined and the position corresponding to the bubble count closest to their average was assigned as $50 \%$ drop. The proton range in the phantom was then compared with the end of the vaporization zone, defined as the position at which the bubble count drops by $50 \%$.

To evaluate the accuracy of the proposed algorithm to detect the actual position of the $50 \%$ drop in bubble count, 1000 artificial vaporization maps (figure $3(\mathrm{~b})$ ) for which the positions of the irradiated zones were fixed were generated and tested. In particular, bubble densities were derived from the experimental measurements for each dose $(0,2,10$ and 20 Gy). The artificial vaporization maps were fed to the image processing algorithms (steps (c) to (f) of figure 3 ) and the $50 \%$ drop position was deduced. In order to quantify the uncertainties introduced by the image processing steps, we characterized the error between the obtained 50\% drop position $(n=1000)$ and the known position of the transition zone (between vaporized and non-vaporized areas) in the artificial vaporization maps.

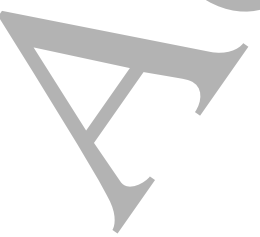

\subsection{Statistical analyses}

Statistical data were calculated as mean \pm standard deviation. Differences in bubble count between the three ROIs (forward, reverse and central distal regions) in phantoms of the same condition (irradiated or control) were examined using two-tailed Student's t-tests. Increases in bubble count between images acquired before and after irradiation were assessed for control and irradiated samples using one-tailed paired Student's t-tests on the corresponding ROIs. Finally, the difference in bubble count increase between irradiated and control groups was evaluated with a one-tailed Student's ttest. All tests were performed in Matlab (R2018b, The Mathworks, Natick, MA, USA) with a significance level $(\alpha)$ of 0.05 .

\section{Results}

\subsection{Analytical evaluation of the experimental conditions}

In this study, the radiation response of nanodroplets with a decafluorobutane core at $25^{\circ} \mathrm{C}$ was evaluated. The necessary physical quantities to estimate the required vaporization energies are listed in table 2 . Using these values in equations (1-5) results in a nucleation energy $W_{\text {tot }}$ of $66 \mathrm{keV}$ and a 
Table 2. Physical properties of $\mathrm{C}_{4} \mathrm{~F}_{10}$ at $25^{\circ} \mathrm{C}$

\begin{tabular}{lcc}
\hline Property & Symbol & Value [unit] \\
\hline Surface tension & $\sigma$ & $7.19 \times 10^{-3}\left[\mathrm{~N} \mathrm{~m}^{-1}\right]$ \\
Saturation pressure & $p_{s}$ & $2.68 \times 10^{5}[\mathrm{~Pa}]$ \\
Latent vaporization heat & $\Delta H$ & $8.75 \times 10^{4}\left[\mathrm{~J} \mathrm{~kg}^{-1}\right]$ \\
Liquid pressure & $p_{l}$ & $1.01 \times 10^{5}[\mathrm{~Pa}]$ \\
Heat conductivity & $k$ & $4.27 \times 10^{-2}\left[\mathrm{~W} \mathrm{~m}^{-1} \mathrm{~K}^{-1}\right]$ \\
Gas density & $\rho_{v}$ & $2.89 \times 10^{-2}\left[\mathrm{~kg} \mathrm{~m}^{-3}\right]$ \\
Liquid density & $\rho_{l}$ & $1.50\left[\mathrm{~kg} \mathrm{~m}^{-3}\right]$ \\
Specific heat capacity & $c_{p}$ & $1.08 \times 10^{3}\left[\mathrm{~J} \mathrm{~kg}^{-1} \mathrm{~K}^{-1}\right]$ \\
\hline
\end{tabular}

critical radius $R_{c}$ of $89 \mathrm{~nm}$. In order to compute the LET threshold from the semi-empirical nucleation theory, we set the nucleation parameter equal to two, in agreement with the experimental findings of d'Errico (D'Errico 1999) for moderate $(<<1 \mathrm{MeV})$ values of the critical nucleation energy $W_{t o t}$. This led to a calculated track-averaged LET threshold of $370 \mathrm{keV} / \mu \mathrm{m}$. At $25^{\circ} \mathrm{C}$, the reduced superheat value of the nanodroplet dispersion was 0.23 .

\subsection{Radiation response of the nanodroplet formulation}

Examples of ultrasound images aligned to the center of the phantom are displayed in figure $4(\mathrm{a}-\mathrm{f})$. Before irradiation, the two phantoms with dispersed nanodroplets are similar, with only a few visible microbubbles (figure 4(c,e)). The microbubbles appear bright due to the large acoustic impedance mismatch between the surrounding waterequivalent matrix and the microbubble gaseous core. On the contrary, the liquid core of the nanodroplets is invisible on the ultrasound images. As microbubbles are too small to be resolved by ultrasound imaging, their shape on the image is dictated by the point spread function of the ultrasound system. The irradiated phantoms (figure 4(d)) exhibit spatially confined zones of higher bubble count inside the primary proton beam path compared to the area beyond the Bragg peak (middle zone). The number of bubbles is higher for the $20 \mathrm{~Gy}$ region compared to $10 \mathrm{~Gy}$. After immersion at $25^{\circ} \mathrm{C}$, the control phantoms (figure 4(f)) displayed) an increased, homogeneous bubble density similar to the one observed in the middle zone of the irradiated phantoms. No bubbles were detected before or after irradiation in the phantom without nanodroplets (figure $4(a-b)$ ).

Bubble signals were counted as described in section 2.6.1 and a typical identification result is displayed in figure 5. Afterwards, phantoms were grouped per condition, irradiated with $62 \mathrm{MeV}$ protons in forward and reverse positions $(n=3$, pre and post) and control ( $n=3$, pre and post). Potential differences between the three ROIs (spatial differences in bubble density) were assessed with Student's t-tests for each condition. Only the irradiated group, post-irradiation, exhibited a statistically significant difference in bubble count between the three zones $(p<0.05)$. This confirmed the homogeneous dispersion of the droplets in the phantom, as the bubble density in non-irradiated samples was spatially uniform. Then, the increase in bubble count between pre and post images for irradiated and control groups was evaluated

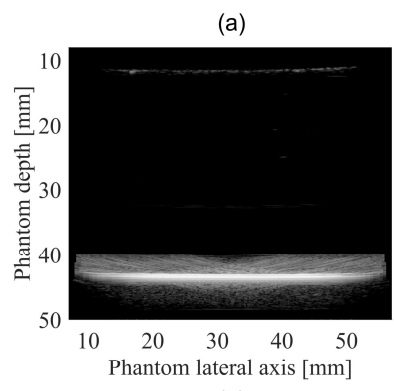

(b)

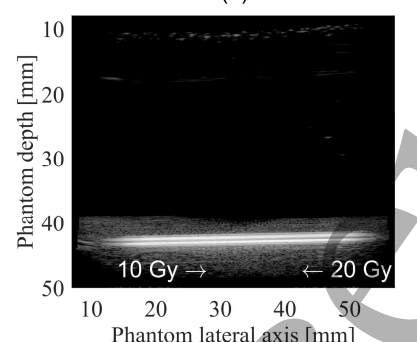

Phantom lateral axis $[\mathrm{mm}]$ (c)

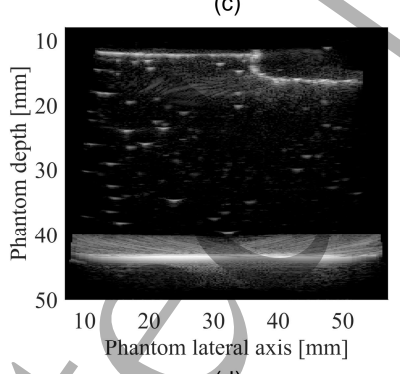
(d)

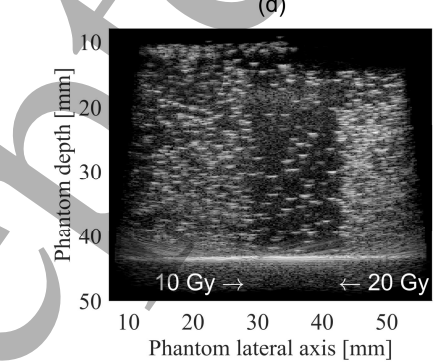

(e)

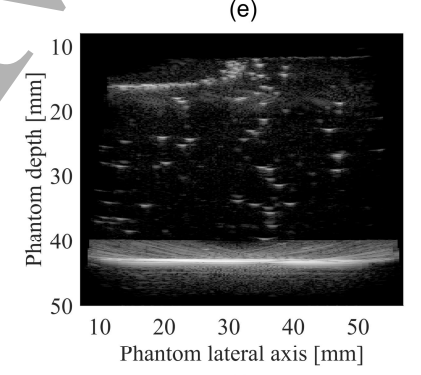

(f)

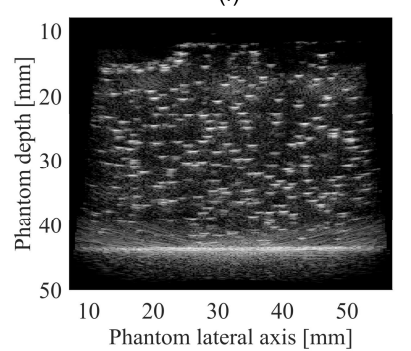

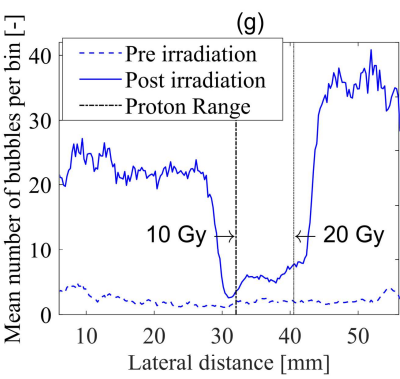

(h)

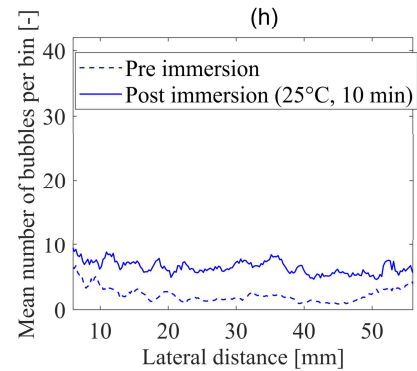

Figure 4. Ultrasound images of a phantom made of pure gelatin before (a) and after (b) exposure to $62 \mathrm{MeV}$ protons (10 Gy dose delivered in the forward position, $20 \mathrm{~Gy}$ in the reverse position). Gelatin phantom with dispersed nanodroplets before (c) and after (d) an identical irradiation scheme. Corresponding images for a control phantom with dispersed nanodroplets before (e) and after (f) immersion at $25^{\circ} \mathrm{C}($ no irradiation). (g) Bubble count profile across the irradiated phantom with nanodroplets (c-d), averaged over 11 imaging frames. (h) Bubble count profile for the control phantom with nanodroplets (e-f). 


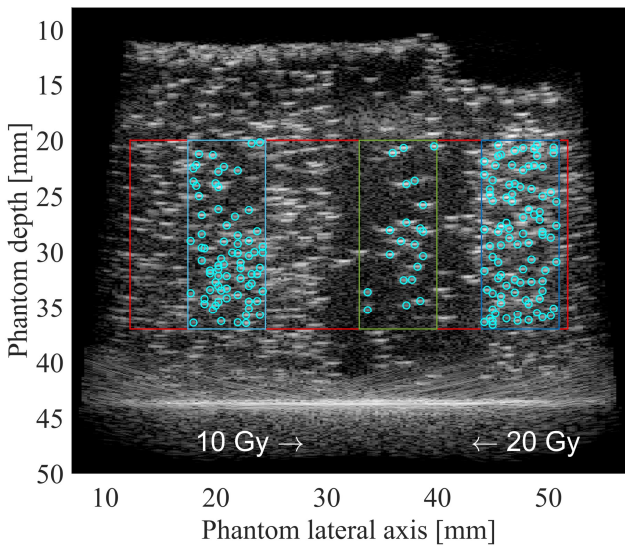

Figure 5. Delineation of regions of interest in the two irradiated regions and in the zone distal to both Bragg peaks, on a post-irradiation ultrasound image. The round markers indicate bubble counts. The vaporization profiles across the image lateral axis are computed over the red region of interest.

with a one-tailed paired t-test. For both irradiated and control groups, we observed a significant increase $(\mathrm{p}<0.05)$ in bubble count, indicating that all phantoms exhibit a certain degree of spontaneous vaporization over time. Finally, we investigated whether the bubble count increase was more pronounced in the irradiated group. The mean and standard deviation of the difference in bubble count between images acquired before and after irradiation are displayed in figure 6 for each ROI of the control and irradiated groups. Statistically significant $(p<0.01)$ differences in bubble count were observed between irradiated and control phantoms for the left and right ROIs, corresponding to the $10 \mathrm{~Gy}$ and 20 Gy zones, respectively. No significant difference between irradiated and control groups was found for the zone distal to the Bragg peaks.

\subsection{Proton range verification}

Lateral bubble count profiles (figure $4(\mathrm{~g}-\mathrm{h})$ ) were derived from the ultrasound images (figure 4(c-f)) as explained in section 2.6.2. Figure 7 shows the profile of two individual phantoms irradiated with $62 \mathrm{MeV}$ and $46.8 \mathrm{MeV}$ protons, as well as the corresponding absolute range measurements. For

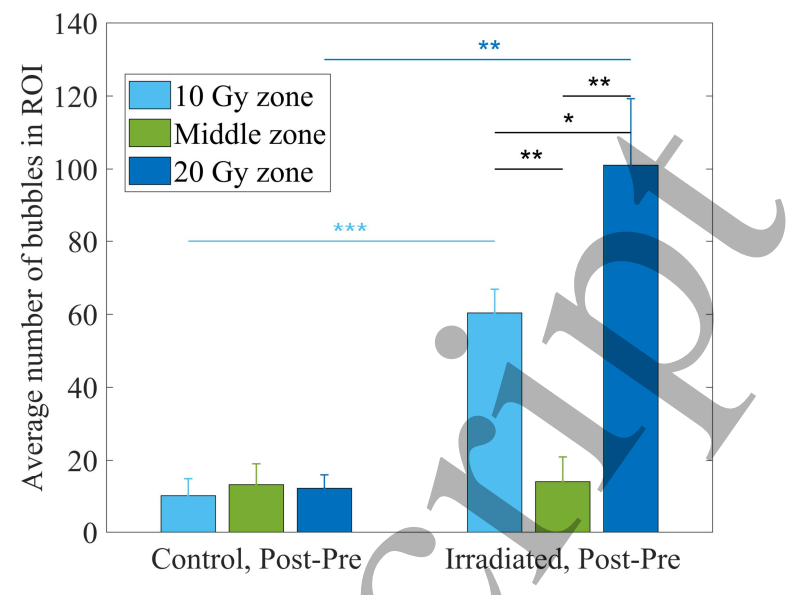

Figure 6. Difference between post-irradiation and pre-irradiation bubble count in the three ROIs, for the irradiated $(n=3,62 \mathrm{MeV})$ and control (no irradiation) group $(n=3)$. Statistical significance is indicated by the presence of stars: $\mathrm{p}<0.05(*), \mathrm{p}<0.01(* *)$ and $\mathrm{p}<0.001$ $(* * *)$.

both beam energies, the bubble count profiles did not follow the characteristic Bragg profile, but instead appeared as step functions, with higher bubble counts for 20 Gy irradiations compared to 10 Gy (figure 4(g)). Additionally, for each phantom, the bubble count abruptly dropped a few millimeters proximal to the dose maximum position.

Signal shifts, calculated as the difference between the $R_{80}$ value obtained from the absolute range measurements and the position corresponding to a 50\% drop in bubble count (star in figure 7), are listed in table 3 together with the measurement uncertainties estimated as described in section 2.6. One of the phantoms irradiated with $46.8 \mathrm{MeV}$ was discarded due to high background signal (elevated by $9 \mathrm{~dB}$ with respect to other phantoms), hampering profile extraction. To compensate for this, an additional phantom containing $6 \%$ gelatin and $25 \mu \mathrm{M}$ nanodroplets (not included in table 1), was irradiated with 46.8 $\mathrm{MeV}$ protons during a follow-up experiment. A signal shift of $2.79 \pm 0.26 \mathrm{~mm}$ was obtained for irradiation with $62 \mathrm{MeV}$ protons and a shift of $2.98 \pm 0.22 \mathrm{~mm}$ was observed for 46.8 $\mathrm{MeV}$ protons. However, due to measurement variability and uncertainty on the $50 \%$ drop position, we cannot establish

Table 3. Signal shifts experimentally determined for six irradiated phantoms and estimated measurement uncertainties. The bubble localization error was determined by comparing the in-house developed algorithm with super-resolution techniques. The error on the position of the $50 \%$ drop in bubble count was estimated from artificial vaporization maps $(n=1000)$.

\begin{tabular}{|c|c|c|c|c|}
\hline Phantom & Beam Energy & Signal shift & $\begin{array}{c}\text { Bubble localization } \\
\text { error }\end{array}$ & $\begin{array}{c}\text { Error on the bubble count drop localization } \\
\text { Mean } \pm \text { standard deviation }\end{array}$ \\
\hline $\begin{array}{l}6 \% \text { Gelatin } \\
25 \mu \mathrm{M} \text { NDs }\end{array}$ & $\begin{array}{l}62 \mathrm{MeV} \\
62 \mathrm{MeV} \\
62 \mathrm{MeV}\end{array}$ & $\begin{array}{l}2.87 \mathrm{~mm} \\
3.00 \mathrm{~mm} \\
2.50 \mathrm{~mm}\end{array}$ & $<0.25 \mathrm{~mm}$ & $0.11 \pm 0.09 \mathrm{~mm}$ \\
\hline $\begin{array}{l}6 \% \text { Gelatin } \\
50 \mu \mathrm{M} \text { NDs }\end{array}$ & $\begin{array}{l}46.8 \mathrm{MeV} \\
46.8 \mathrm{MeV}\end{array}$ & $\begin{array}{l}3.11 \mathrm{~mm} \\
3.11 \mathrm{~mm} \\
\end{array}$ & $<0.25 \mathrm{~mm}$ & $0.34 \pm 0.39 \mathrm{~mm}$ \\
\hline $\begin{array}{l}6 \% \text { Gelatin } \\
25 \mu \mathrm{M} \text { NDs }\end{array}$ & $46.8 \mathrm{MeV}$ & $2.73 \mathrm{~mm}$ & $<0.25 \mathrm{~mm}$ & $0.32 \pm 0.37 \mathrm{~mm}$ \\
\hline
\end{tabular}




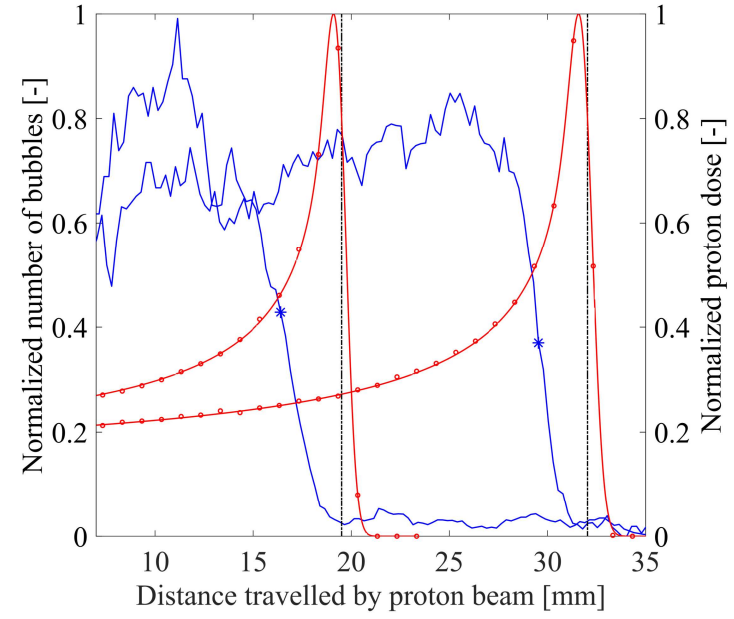

Figure 7. Comparison of bubble vaporization profiles along the lateral direction of ultrasound images (parallel to the proton beam) and measured proton dose deposition with depth, for delivered doses of $10 \mathrm{~Gy}$ and beam energies of 62 and $46.8 \mathrm{MeV}$. The position of the $50 \%$ drop in bubble count is marked by a star, and the dotted vertical lines represent $R_{80}$ values.

whether there is a difference between the signal shifts observed with $62 \mathrm{MeV}$ and $46.8 \mathrm{MeV}$ protons. The distance between the end of the dense bubble region and the proton range was measured with sub-millimeter repeatability for both energies.

\subsection{Nanodroplet sensitivity to proton dose and fluence}

After evaluating the radiation response for large proton doses (10 and $20 \mathrm{~Gy}$ ), we assessed whether the same results could also be obtained for clinically-relevant doses. Since less vaporization events were expected, the droplet concentration in the phantoms was doubled. An ultrasound image acquired after delivery of a 2 Gy dose in the Bragg peak with $62 \mathrm{MeV}$ protons is displayed in figure 8 . Again, a distinct zone of high bubble density was observed proximal to the Bragg peak, confirming the capability of the superheated nanodroplets to detect clinically-relevant doses.

The relationship between the dose and the resullting bubble counts is depicted in figure 9 . As before, isometric ROIs were defined in zones irradiated with 2,10 and $20 \mathrm{~Gy}$ as well as the corresponding zones distal to the Bragg peak. The latter served as internal reference and the resulting bubble counts were subtracted from the values obtained from the irradiated zones to account for spontaneous droplet vaporization. The 0 Gy data points were analogously obtained from the control phantoms. A linear regression line was fitted through the 0,10 and $20 \mathrm{~Gy}$ data points, which were all acquired for the same droplet concentration. Bubble counts for the 2 Gy irradiation were rescaled by a factor of 0.5 to account for the double droplet concentration. However, since we ignore potential concentration dependent effects, these data points were not used for curve fitting. Nevertheless, the $2 \mathrm{~Gy}$ bubble counts were located well within the calculated confidence interval of the fit.

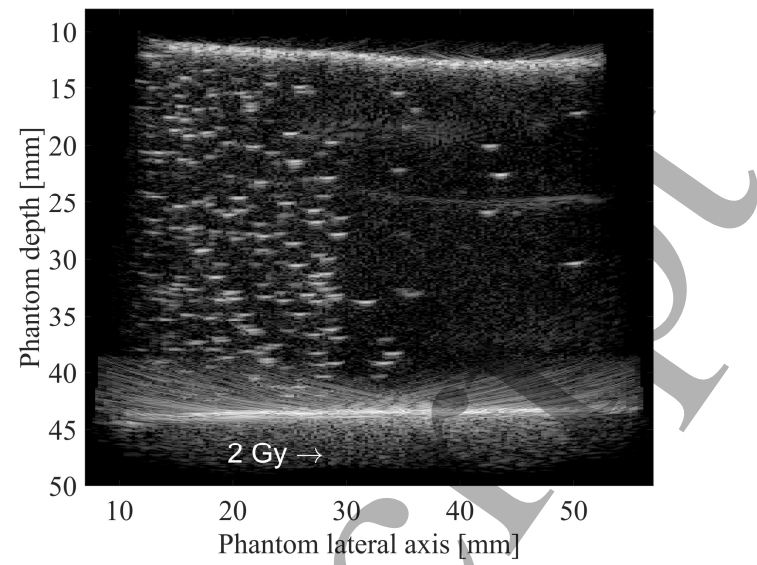

Figure 8. Ultrasound image acquired after phantom irradiation with clinically-relevant parameters (dose: $2 \mathrm{~Gy}$, dose rate: $2 \mathrm{~Gy} / \mathrm{min}$ and beam energy of $62 \mathrm{MeV}$ )

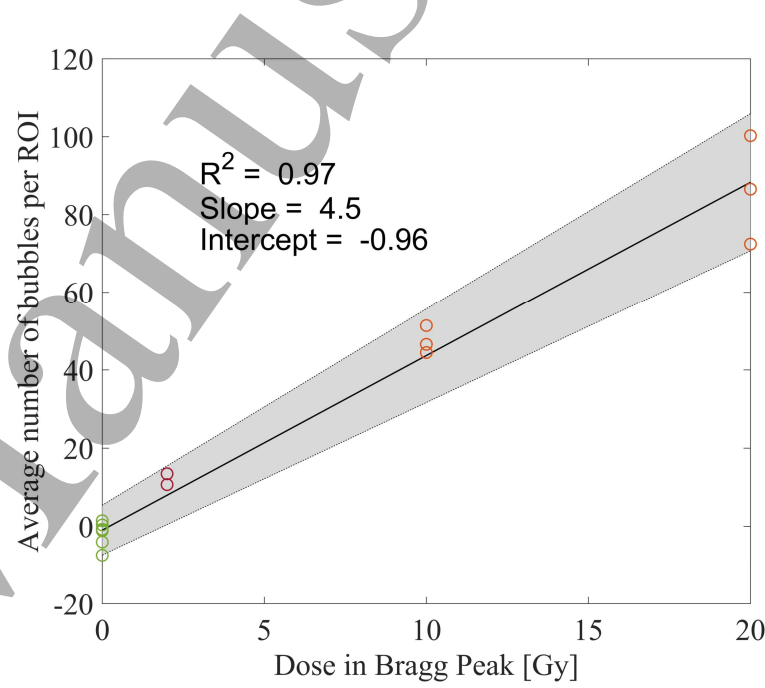

Figure 9. Evolution of the number of vaporization events with proton dose. Circles represent experimental data, and the black line is the linear regression fit. The $95 \%$ confidence interval on the linear regression is shown by the shaded area.

\section{Discussion}

In this study, we evaluated the radiation sensitivity of submicron-sized superheated droplets in proton beams, assessing their potential for proton dosimetry and range verification. First, we verified the radiation response of the gelatin matrix in terms of ultrasound contrast generation. As displayed in figure $4(a-b)$, the gelatin phantom without nanodroplets resulted in a background-free ultrasound image after irradiation, demonstrating that the radiation-induced contrast generation was attributed to the presence of nanodroplets. The background signal present in phantoms before irradiation (figure 4(c,e)) was due to spontaneous vaporization of a small fraction of superheated nanodroplets either already in the nanodroplet vial or during phantom 
preparation. The increase in bubble count was significantly higher for irradiated phantoms, in regions proximal to the proton range, compared to non-irradiated phantoms, confirming that the observed response (figure 4(d)) is induced by proton irradiation.

The theoretical threshold LET value for droplet vaporization was determined to be $370 \mathrm{keV} / \mu \mathrm{m}$. Hence, sensitivity to neither the primary proton beam (exhibiting a maximum LET of $70-90 \mathrm{keV} / \mu \mathrm{m}$ at the distal end of the Bragg peak) nor to secondary alpha particles (LET ranging from 130 to $190 \mathrm{keV} / \mu \mathrm{m}$ ) (Grassberger and Paganetti 2011) was expected. This was confirmed by the nearly flat bubble count profiles observed, with no enhancement in the Bragg peak location, which would have been observed for both protons and alpha particles. Instead, we hypothesize that bubble vaporization was caused by nuclear recoils, whose LET can range from several hundreds to a thousand $\mathrm{keV} / \mu \mathrm{m}$ (Grassberger and Paganetti 2011), created from interactions with either the primary proton beam or secondary neutrons. However, the contribution of the latter was assumed negligible as we did not observe a significant increase of bubbles in the region distal to the Bragg peak.

To confirm that bubble vaporization was induced by recoil ions, we extracted nuclear reaction cross sections of $\mathrm{C}, \mathrm{N}, \mathrm{O}$ and $\mathrm{F}$ (atoms present in the gelatin matrix and nanodroplets) from the TENDL-2014 database, which rely on the advanced nuclear reaction simulation software TALYS (Koning et al 2014), and displayed them together with a bubble count profile in figure 10(a). The average proton energy at each depth was determined based on the PSTAR (Berger et al 2005) residual continuous slowing down approximation (CSDA) range in water and used to evaluate the reaction cross section at these positions. For each atom, the reaction cross section drops similarly to the bubble profile - proximal to the Bragg peak. This is due to the Coulomb barrier of the nucleus, which has to be overcome for a non-elastic nuclear reaction to take place (Newhauser and Zhang 2015). For oxygen, this threshold energy is $8 \mathrm{MeV}$, which corresponds to a residual range in water of $0.83 \mathrm{~mm}$. In section 3.3 , the distance between the $50 \%$ drop in bubble count and the range of $62 \mathrm{MeV}$ protons measured in water was estimated to be $2.79 \pm 0.26 \mathrm{~mm}$. We measured a difference of $1.8 \%$ in density between pure water and a $6 \%$ gelatin matrix, leading to a CSDA range decrease of $0.59 \mathrm{~mm}$ (Berger et al 2005) that is accounted for in figure 10 . However, a small discrepancy between the steep drop in oxygen recoil production and the experimentally determined vaporization profile remains. This indicates that the presence of recoil nuclei is insufficient for droplet vaporization. Indeed, superheated drop detectors are LET-dependent, and the maximal amount of energy transferred to recoil nuclei decreases with the energy of incident protons (Seltzer 1993). Therefore, we also evaluated the track-averaged LET of oxygen recoils produced along the phantom depth. The average energy transferred to heavy recoils $(A>4)$ from proton-oxygen nuclear interactions was extracted from the ICRU report $n^{\circ} 63$ (ICRU 2000). For these energies, the range of an oxygen ion in water with a density of $1.018 \mathrm{~g} / \mathrm{cm}^{3}$ was determined with SRIM (Ziegler 2013) and used to calculate the track-averaged LET. The result is depicted in figure 10(b), together with the theoretical threshold of $370 \mathrm{keV} / \mu \mathrm{m}$, and a bubble count profile. The depth at which the track-averaged LET of oxygen recoils drops below the LET threshold

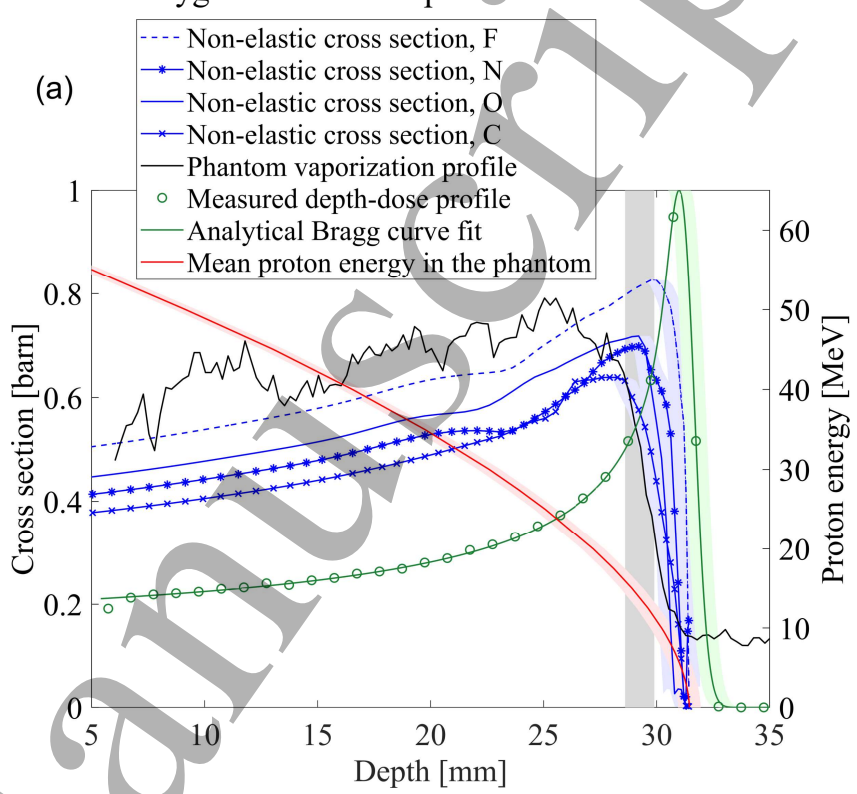

(b) - Phantom vaporization profile

Measured depth-dose profile

1 - Analytical Bragg curve fit
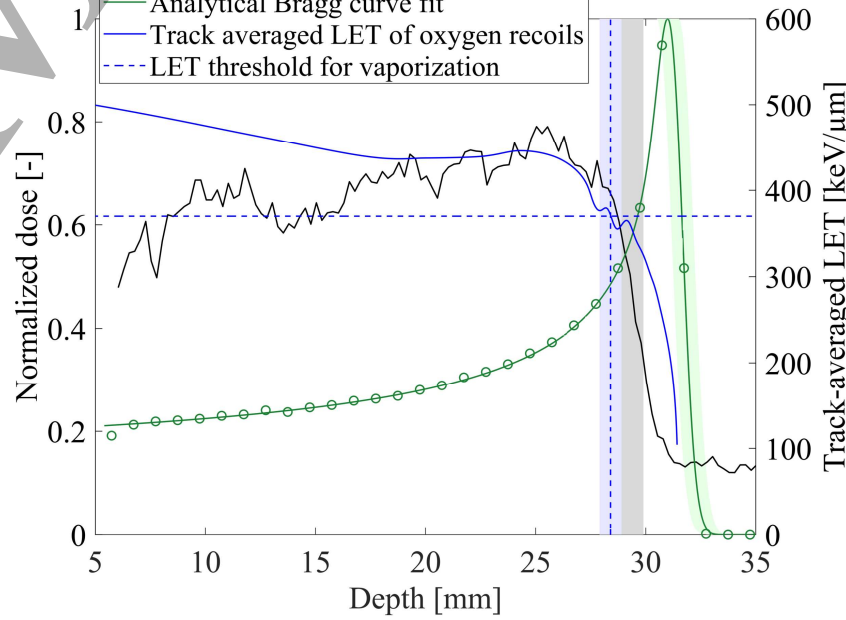

Figure 10. (a) Overlay of the droplet vaporization profile in one phantom with the non-elastic nuclear interaction cross sections for relevant atoms and the proton depth-dose profile. (b) Overlay of the droplet vaporization profile in the phantom with the average trackaveraged LET of oxygen recoils and the proton depth-dose profile. The shaded area surrounding the Bragg peak represents measurement uncertainties on the proton range, which propagate to uncertainties on the residual proton energy in the phantom (in red) and on the non-elastic cross section and track-averaged LET estimates (in blue). Uncertainties on the position of the $50 \%$ drop in bubble count are represented by the gray area. 
coincides with the start of the drop in bubble count. The latter provides evidence that only recoils of sufficient LET can trigger nanodroplet vaporization. This is in agreement with the radiation-induced nucleation theory (section 2.1). Moreover, this can also explain why no bubbles were detected at or closely in front of the Bragg peak (figure 10). The measurement uncertainties are displayed in figure 10(a) and (b) as shaded areas. Uncertainties in the absolute range position arise from potential measurement and fitting errors, whose combined effect was estimated to be less than \pm 0.5 $\mathrm{mm}$. This uncertainty propagates towards the estimated position of the non-elastic cross sections and track-averaged LET of recoil ions, which depend on the residual proton energy. The $95 \%$ confidence interval in the position of the drop in bubble count, extracted from the measured signal shifts ( $n=3$, see table 3 ) observed in phantoms irradiated with $62 \mathrm{MeV}$ protons, is represented as a gray area. Furthermore, longitudinal range straggling of $62 \mathrm{MeV}$ protons in water was simulated using TRIM (Ziegler 2013) and determined to be $508 \mu \mathrm{m}$. As the initial energy dispersion of the proton beam is unknown, we did not account for range straggling in figure 10. Despite the aforementioned uncertainty arising from the limited resolution of the experimental measurements, these results suggest that the features of the observed bubble count profiles can be related to the non-elastic reaction cross section and the energy deposition characteristics of oxygen recoils This supports the hypothesis that the radiation-induced nucleation theory is applicable to droplets of nanometer size.

The transition from drops of several microns, commonly used in superheated drop detectors, to nanometer-sized droplets has two important implications. Firstly, the assumption that the recoil ions responsible for droplet vaporization are only formed within the superheated liquid (D'Errico 2001) no longer holds, as the nanodroplet diameters are several times smaller than the mean range of recoil ions. Hence, both recoils produced inside the droplets and in the surrounding gelatin matrix can induce droplet vaporization. For this reason, we considered oxygen as the dominant recoil ion, given its relative abundance in the phantom matrix. Secondly, while negligible in micro-emulsions, the contribution of the Laplace pressure to the pressure inside the droplet $\left(p_{l}\right)$ becomes important for small droplet radii as described in equation 9.

$$
\Delta P_{\text {lap }}=\frac{2 \gamma}{R}
$$

Here, $\gamma$ denotes the surface tension at the droplet interface and therefore depends on the polymerized PCDA layer. A positive Laplace pressure will decrease the degree of superheat of the droplets, and hence raise the LET threshold for droplet vaporization. Since we did not experimentally measure the surface tension, nor could values be adapted from the literature, we did not account for the Laplace pressure. Theoretical considerations and experimental observations support the assumption of a negligible surface tension. Indeed, the nanodroplets employed in this study have an outstanding in-vial stability (Toumia et al 2019), while models predict a fast dissolution of nanodroplets with a positive surface tension (Mountford and Borden 2016). Additionally, it has been described that perfluorocarbons can decrease the overall surface tension of droplets (Unger and Matsunaga 2010). Moreover, due to the large polydispersity of the droplets used (polydispersity index of 0.25 ), it is reasonable to assume that part of the population (i.e., the large droplets) will be relatively unaffected by the Laplace pressure, while only the smallest droplets might experience a decreased superheat.

In addition to proton range verification, we also evaluated whether the bubble count profiles were correlated with the radiation dose. No direct sensitivity to the primary proton beam was established due to the limited degree of superheat of the nanodroplets. Nevertheless, a linear relationship between bubble counts and irradiation dose was obtained for high proton doses of 10 and $20 \mathrm{~Gy}$. We explain this relationship by the fact that the number of nuclear reactions and thus recoil ions is dependent on the proton fluence. As a rule of thumb, the primary proton fluence decreases by $1 \%$ for every centimeter of tissue traversed (Durante and Paganetti 2016) due to nuclear reactions. Hence, for the limited size of the ROIs $(7 \mathrm{~mm})$ along the proton beam, the fluence can be assumed constant. To double the dose from 10 to $20 \mathrm{~Gy}$, the fluence was also doubled, which is captured by the linear relationship in figure 9. This linear response tends to hold for clinically-relevant doses (2 Gy), although further work is required to assess the validity of this relationship at smaller fluences.

Consequently, the experimental findings presented in this contribution show that superheated nanodroplets can provide indirect information on the proton range and fluence, by generating ultrasound contrast upon interaction with highLET nuclear recoils. The latter provides one of the major advantages of the presented approach over current state-ofthe-art range verification tools such as PGI and PET imaging, which are also based on nuclear reactions. Since this technique is not relying on specific reaction channels, like prompt gamma emitting channels or channels in which positron emitting isotopes are generated, it has the potential to detect a larger number of nuclear events. Moreover, the detection of nuclear recoils via individually detectable microbubbles provides a strong, inherent signal enhancement.

To accurately relate the observed signals to the primary proton beam, Monte Carlo (MC) simulations are required. Apart from providing a means for in vivo range verification, these could potentially be employed for in vivo proton dosimetry by taking advantage of the fluence dependency. To assess this hypothesis and better understand the LET vaporization thresholds, we are currently implementing comprehensive MC simulations describing individual 
nanodroplets as detectors. However, MC simulations of nuclear interactions suffer from uncertainties arising from the limited amount of experimental data available to describe interaction cross sections for biologically-relevant targets (Paganetti 2012).

Alternatively, increasing the degree of superheat to sensitize nanodroplets to the primary proton beam might be beneficial, as the vaporization profiles could be directly related to the proton range and dose distribution. Additionally, nuclear recoils only represent a very small percentage of all interactions, leading to a low fraction of nanodroplets undergoing vaporization. For in vivo applications, a high yield might be recommended to minimize the required droplet concentration and related potential side-effects. However, highly superheated droplets will be more prone to spontaneous vaporization. Hence, the appropriate choice of degree of superheat will depend on the achievable signal-to-noise ratio.

One could argue that the temperature of $25^{\circ} \mathrm{C}$ chosen for this study is unsuitable as it does not fully reflect the response of the nanodroplets at physiological temperature, due to the high dependence of the radiation response on the degree of superheat. At $37^{\circ} \mathrm{C}$, the reduced degree of superheat for $\mathrm{C}_{4} \mathrm{~F}_{10}$ droplets, $s=0.33$, is theoretically still insufficient to expect a response to the primary proton beam. However, the proton range would have to be inferred from vaporization profiles related to secondary radiation products, comprised not only of heavy recoil ions, but potentially also of light secondaries such as alpha particles and deuterons, as the track-averaged LET threshold would drop to $145 \mathrm{keV} / \mu \mathrm{m}$. Moreover, the composition of the nanodroplets superheated liquid core can be altered to appropriately tune the degree of superheat to the desired LET thresholds. Indeed, a variety of perfluorocarbons with different boiling points have been employed to formulate nanodroplets (Sheeran et al 2017).

The use of ultrasound imaging to noninvasively evaluate vaporization events has numerous advantages for clinical applications such as its inexpensiveness, small footprint, portability, short examination times and real-time capabilities. In our study, we did not employ an ultrasound pulse sequence tailored for microbubble contrast agent imaging, and the limited image quality and resolution affected the accuracy of the estimation of the absolute position of the drop in bubble count. Additionally, the algorithm developed for bubble detection was not optimal for zones of high bubble density. To verify that the bubble counting algorithm did not introduce any bias in the results, we compared it with results obtained by considering the mean gray value as the relevant metric for vaporization (instead of the number of bubbles) and found no noticeable difference. In order to benefit from the improved ultrasound image resolution in the axial direction, the axial ultrasound axis should coincide with the proton beam direction. Further research should be conducted to obtain the optimal droplet concentration and maximize the contrast between irradiated and non-irradiated regions.

Proton irradiations were performed in an experimental research facility, with a passively-scattered monoenergetic beam whose characteristics differ from clinical proton beams. In particular, the features of the vaporization profiles, with a steep drop in front of the proton range, might be altered in a spread-out Bragg peak. Future studies aim to establish the response of the superheated nanodroplets in clinical proton beams, including scanned beams.

\section{Conclusion}

In this contribution, the potential use of nanodroplets for proton dosimetry and range verification was investigated. Downscaling radioresponsive micro-emulsions used in superheated drop detectors to injectable phase-change contrast agents produced ultrasonically detectable radiation sensors with potential for in vivo use, provided that a similar radiation response would be observed under clinical proton therapy conditions. Applying radiation-induced nucleation theory to our experimental conditions revealed that the decafluorobutane liquid core vaporizes when exposed to high-LET secondary particles generated during nuclear reactions of the proton beam. Nanodroplet dispersions exposed to monoenergetic proton beams of $62 \mathrm{MeV}$ and $46.8 \mathrm{MeV}$, at $25^{\circ} \mathrm{C}$, exhibited spatially confined bubble vaporization regions proximal to the Bragg peak. While ultrasound signals dropped before the actual proton range due to LET dependencies, the resulting signal shift was determined with sub-millimeter precision. Additionally, the bubble count was linearly related to the proton fluence. Lastly, the potential of the developed technique was shown at a clinically-relevant dose of 2 Gy. Future work aims to confirm these early findings and refine the range estimates accuracy. Proton irradiations at $37^{\circ} \mathrm{C}$ will be carried out to assess the relevance of these proof-of-concept data at physiological temperatures. Finally, the nanodroplet design will be optimized to ensure sensitivity to the primary proton beam, enabling direct in vivo proton range verification and potentially in vivo proton dosimetry.

\section{Acknowledgements}

The authors would like to acknowledge the team of the "Centre de Ressources du Cyclotron" of UCLouvain for their precious assistance in operating the proton beam and performing absolute range measurements. The authors also thank Fraunhofer IBMT for providing the ultrasound readout system, and the mechanical and electrical staff of the Physics Department of KU Leuven for the design and conception of the water tank heating system and phantom containers. This work has been supported by the European Union's Horizon 2020 research and innovation programme under grant agreement $n^{\circ} 766456$ (“AMPHORA”). BC received a $\mathrm{PhD}$ 
fellowship fundamental research from the Research Foundation Flanders (n¹1A9520N).

\section{References}

Andrews H R, Noulty R A, Ing H, d'Errico F, Lewis B J, Bennett L G I and Green A R 2006 LET dependence of bubble detector response to heavy ions Radiat. Prot. Dosimetry 120 480-4

Apfel R E 1998 Activatable infusable dispersions containing drops of a superheated liquid for methods of therapy and diagnosis US Patent no. 5,840,276

Apfel R E 1979 The superheated drop detector Nucl. Instruments Methods 162 603-8

Berger M J, Coursey J S, Zucker M A and Chang J 2005 ESTAR, PSTAR and ASTAR: Computer Programs for Calculating Stopping-Power and Range Tables for Electrons, Protons and Helium Ions (version 2.0.1) Natl. Inst. Stand. Technol. Gaithersburg, MD. Online: https://physics.nist.gov/PhysRefData/Star/Text/PSTAR.html

Bortfeld T 1997 An analytical approximation of the Bragg curve for therapeutic proton beams Med. Phys. 24 2024-33

Culjat M O, Goldenberg D, Tewari P and Singh R S 2010 A review of tissue substitutes for ultrasound imaging Ultrasound Med. Biol. 36 861-73

D'Errico F 1999 Fundamental properties of superheated drop (bubble) detectors Radiat. Prot. Dosimetry 84 55-62

D'Errico F 2001 Radiation dosimetry and spectrometry with superheated emulsions Nucl. Instruments Methods Phys. Res. Sect. B Beam Interact. with Mater. Atoms 184 229-54

D’Errico F, Apfel R E, Curzio G, Dietz E, Egger E, Gualdrini G F, Guldbakke S, Nath R and Siebert B R L 1997 Superheated emulsions: neutronics and thermodynamics Radiat. Prot. Dosimetry 70 109-12

D'Errico F and Egger E 1994 Proton beam dosimetry with superheated drop (bubble) detectors Hadrontherapy in Oncology: Proceedings of the First International Symposium on Hadrontherapy (Como, Italy) ed U Amaldi and B Larsson (Amsterdam: Excerpta Medica, International Congress Series 1077, Elsevier) pp 488-94

D'Errico F, Nath R and Nolte R 2000 A model for photon detection and dosimetry with superheated emulsions Med. Phys. 27 401-9

Deshpande N, Needles A and Willmann J K 2010 Molecular ultrasound imaging: current status and future directions Clin. Radiol. 65 567-81

Dove J D, Mountford P A, Murray T W and Borden M A 2014 Engineering optically triggered droplets for photoacoustic imaging and therapy Biomed. Opt. Express $\mathbf{5} 4417$

Durante M and Paganetti H 2016 Nuclear physics in particle therapy: A review Reports Prog. Phys. 79

Glaser D A 1952 Some effects of ionizing radiation on the formation of bubbles in liquids Phys. Rev. 87665

Grassberger C and Paganetti H/2011 Elevated LET components in clinical proton beams Phys. Med. Biol. 56 6677-91

Green A R, Andrews H R, Bennett L G I, Clifford E T H, Ing H, Jonkmans G, Lewis B J, Noulty R A and Ough E A 2005 Bubble detector characterization for space radiation Acta Astronaut. 56 949-60

Guo S L, Li L, Chen B L, Doke T, Kikuchi J, Terasawa K, Komiyama M, Hara K and Fuse T 2002 Proton tracks in bubble detector Nucl. Instruments Methods Phys. Res. Sect. B Beam Interact. with Mater. Atoms 198 135-41

Helmbrecht S, Santiago A, Enghardt W, Kuess P and Fiedler F 2012 On the feasibility of automatic detection of range deviations from in-beam PET data Phys. Med. Biol. 57 138797

ICRU 2000 Nuclear Data for Neutron and Proton Radiotherapy and for Radiation Protection (ICRU Report $n^{\circ} 63$ ) (Bethesda, $\mathrm{MD})$

Ing H, Noulty R A and Mclean T D 1997 Bubble detectors - a maturing technology Radiat. Meas. 27 1-11

Jongen Y and Stichelbaut F 2003 Verification of the proton beams position in the patient by the detection of prompt gammarays emission 39th Meeting of the Particle Therapy CoOperative Group (San Francisco, CA)

Knopf A C and Lomax A 2013 In vivo proton range verification: A review Phys. Med. Biol. 58 131-60

Koning A J, Rochman D, van der Marck S C, Kopecky J, Sublet J C, Pomp S, Sjostrand H, Forrest R, Bauge E, Henriksson H and others 2014 TENDL-2014: TALYS-based evaluated nuclear data library Database available from http//www.talys.eu/tendl-2012

Kraan A C, Battistoni G, Belcari N, Camarlinghi N, Cirrone G A P, Cuttone G, Ferretti S, Ferrari A, Pirrone G, Romano F, Sala P, Sportelli G, Straub K, Tramontana A, Del Guerra A and Rosso V 2014 Proton range monitoring with in-beam PET: Monte Carlo activity predictions and comparison with cyclotron data Phys. Medica 30 559-69

Lea-Banks H, O’Reilly M A and Hynynen K 2019 Ultrasoundresponsive droplets for therapy: A review J. Control. Release 293 144-54

Miller A, Machrafi R, Benton E, Kitamura H and Kodaira S 2018 Comparison of the space bubble detector response to spacelike neutron spectra and high energy protons Acta Astronaut. $1511-6$

Min C H, Kim C H, Youn M Y and Kim J W 2006 Prompt gamma measurements for locating the dose falloff region in the proton therapy Appl. Phys. Lett. $892-5$

Min C H, Zhu X, Winey B A, Grogg K, Testa M, El Fakhiri G, Bortfeld T R, Paganetti H and Shih H A 2013 Clinical Application of in-room PET for in vivo treatment monitoring in proton radiotherapy Int. J. Radiat. Oncol. Biol. Phys. 86 183-9

Mountford P A and Borden M A 2016 On the thermodynamics and kinetics of superheated fluorocarbon phase-change agents Adv. Colloid Interface Sci. 237 15-27

Mumot M, Algranati C, Hartmann M, Schippers J M, Hug E and Lomax A J 2010 Proton range verification using a range probe: Definition of concept and initial analysis Phys. Med. Biol. 55 4771-82

Newhauser W D and Zhang R 2015 The physics of proton therapy Phys. Med. Biol. 60 R155-209

Paganetti H 2019 Proton Therapy Physics (Boca Raton: CRC Press)

Paganetti H 2012 Range uncertainties in proton therapy and the role of Monte Carlo simulations Phys. Med. Biol. 57

Parodi K, Paganetti H, Shih H A, Michaud S, Loeffler J S, DeLaney T F, Liebsch N J, Munzenrider J E, Fischman A J, Knopf A and Bortfeld T 2007 Patient Study of In Vivo Verification of Beam Delivery and Range, Using Positron Emission Tomography and Computed Tomography Imaging After Proton Therapy Int. J. Radiat. Oncol. Biol. Phys. 68 920-34

Plautz T, Bashkirov V, Feng V, Hurley F, Johnson R P, Leary C, Macafee S, Plumb A, Rykalin V, Sadrozinski H F W, Schubert K, Schulte R, Schultze B, Steinberg D, Witt M and Zatserklyaniy A $2014200 \mathrm{MeV}$ proton radiography studies with a hand phantom using a prototype proton CT scanner IEEE Trans. Med. Imaging 33 875-81

Porteous W and Blander M 1975 Limits of superheat and explosive 
boiling of light hydrocarbons, halocarbons, and hydrocarbon mixtures AIchE 21 560-6

Rohling H, Priegnitz M, Schoene S, Schumann A, Enghardt W, Hueso-Gonzalez F, Pausch G and Fiedler F 2017

Requirements for a Compton camera for in vivo range verification of proton therapy Phys. Med. Biol. 622795

Roy S C 2001 Superheated liquid and its place in radiation physics Radiat. Phys. Chem. 61 271-81

Sarkar R, Chatterjee B K, Roy B and Roy S C 2006 Radiation detection by using superheated droplets Radiat. Phys. Chem. $752186-94$

Seitz F 1958 On the theory of the bubble chamber Phys. Fluids 12 13

Seltzer S M 1993 An assessment of the role of charged secondaries from nonelastic nuclear interactions by therapy proton beams in water Natl. Inst. Stand. Technol. Rep. 5221 1-80

Sheeran P S and Dayton P A 2012 Phase-change contrast agents for imaging and therapy. Curr. Pharm. Des. 18 2152-65

Sheeran P S, Luois S H, Mullin L B, Matsunaga T O and Dayton P A 2012 Design of ultrasonically-activatable nanoparticles using low boiling point perfluorocarbons Biomaterials $\mathbf{3 3}$ 3262-9

Sheeran P S, Matsuura N, Borden M A, Williams R, Matsunaga T O, Burns P N and Dayton P A 2017 Methods of generating submicrometer phase-shift perfluorocarbon droplets for applications in medical ultrasonography IEEE Trans. Ultrason. Ferroelectr. Freq. Control 64 252-63

Sheeran P S, Wong V P, Luois S, McFarland R J, Ross W D, Feingold S, Matsunaga T O and Dayton P A 2011 Decafluorobutane as a Phase-Change Contrast Agent for Low-Energy Extravascular Ultrasonic Imaging Ultrasound Med. Biol. 37 1518-30

Takada M, Kitamura H, Koi T, Nakamura T and Fujitaka K 2004 Measured proton sensitivities of bubble detectors Radiat.

\section{Prot. Dosimetry 111 181-9}

Thariat J, Hannoun-Levi J M, Sun Myint A, Vuong T and Gérard J P 2013 Past, present, and future of radiotherapy for the benefit of patients Nat. Rev. Clin. Oncol. 10 52-60

Toumia Y, Cerroni B, Domenici F, Lange H, Bianchi L, Cociorb M, Brasili F, Chiessi E, D'Agostino E, Van Den Abeele K, Heymans S V., D'Hooge J and Paradossi G 2019 Phase Change Ultrasound Contrast Agents with a Photopolymerized Diacetylene Shell Langmuir 35 10116-27

Unger E and Matsunaga T O 2010 Lipid Coated Microbubbles and Nanodroplets as Tools for Biomedical Nanotechnology Handbook of Materials for Nanomedicine vol 1, ed V Torchilin and M M Amiji (Pan Stanford Publishing) pp 74983

Viessmann O M, Eckersley R J, Christensen-Jeffries K, Tang M X and Dunsby C 2013 Acoustic super-resolution with ultrasound and microbubbles Phys. Med. Biol. 58 6447-58

West C D 1998 Cavitation Bubble Nucleation by Energetic Particles (Oak Ridge, Tenessee)

Xie Y, Bentefour E H, Janssens G, Smeets J, Vander Stappen F, Hotoiu L, Yin L, Dolney D, Avery S, O'Grady F, Prieels D, McDonough J, Solberg T D, Lustig R A, Lin A and Teo B K K 2017 Prompt Gamma Imaging for In Vivo Range Verification of Pencil Beam Scanning Proton Therapy Int. J. Radiat. Oncol. Biol. Phys. $99210-8$

Zhang G, Harput S, Lin S, Christensen-Jeffries K, Leow C H, Brown J, Dunsby C, Eckersley R J and Tang M X 2018 Acoustic wave sparsely activated localization microscopy (AWSALM): Super-resolution ultrasound imaging using acoustic activation and deactivation of nanodroplets Appl. Phys. Lett. 113014101

Ziegler J F 2013 SRIM-2013 software package Online: http://www.srim.org 